\section{Como a comunidade internacional da medicina de precisão tem se posicionado diante dos desafios impostos pela pandemia da COVID-19?}

\section{What is the position of the international precision medicine community in relation to the challenges raised by the COVID-19 pandemic?}

\author{
¿Cómo se ha posicionado la comunidad \\ internacional de medicina de precisión \\ ante los desafíos impuestos por la \\ pandemia de COVID-19?
}

Renan Gonçalves Leonel da Silva 1,2 Jorge Alberto Bernstein Iriart 3 doi: 10.1590/0102-311X00296920

\section{Resumo}

Medicina de precisão pode ser definida como um movimento de transformação da biomedicina contemporânea que orienta a atividade de pesquisa acadêmica, modelos de negócios e o desenvolvimento de produtos e serviços de saúde desenhados individualmente para o usuário, baseado em informações genéticas e outros marcadores biomédicos dos pacientes. Ao longo dos últimos anos, essa comunidade tem sido bastante atuante no cenário científico internacional. No entanto, durante a pandemia da COVID-19 ainda não ficou claro quais posicionamentos ou estratégias têm sido adotadas por esses grupos para o enfrentamento da crise sanitária. O objetivo deste artigo é compreender como a comunidade internacional da medicina de precisão está reagindo à pandemia da COVID-19, e em que estão baseadas as suas abordagens e potenciais soluções sugeridas para a mitigação dos efeitos negativos causados pelo aumento das infecções pelo novo coronavírus. Para tanto, foi feita pesquisa documental em 28 documentos provenientes de 18 fontes selecionadas, em que analisou-se as narrativas difundidas pelos profissionais da medicina de precisão em artigos científicos, editoriais, comentários, perspectivas, notícias de jornais e boletins e conferência virtual da Coalizão de Medicina Personalizada (PMC, em inglês). Com isso, buscou-se compreender como esses grupos imaginam uma nova configuração sociotécnica para o enfrentamento da pandemia e de seus efeitos.

Medicina de Precisão; Ciência, Tecnologia e Sociedade; Agenda de Pesquisa em Saúde; COVID-19
Correspondência

R. G. L. Silva

Health Ethics and Policy Lab, Department of Health Sciences and Technology, Eidgenössische Technische Hochschule Zürich. Hottingerstrasse 10, Office: HOA, H17. Zürich 8092,

Switzerland.

leonnelrg@gmail.com

${ }^{1}$ Department of Health Sciences and Technology, Eidgenössische Technische Hochschule Zürich, Zurich, Switzerland.

2 Faculdade de Medicina, Universidade de São Paulo, São Paulo, Brasil.

3 Universidade Federal da Bahia, Salvador, Brasil. 
Ao longo da última década, a chamada medicina de precisão avançou rapidamente no cenário científico e da indústria da saúde internacionalmente. A medicina de precisão pode ser definida como uma abordagem do setor da Saúde que orienta a atividade de pesquisa acadêmica, modelos de negócios e o desenvolvimento de produtos e serviços desenhados individualmente para o usuário, baseado em informações genéticas e outros dados biomédicos dos pacientes. Esse movimento de transformação da biomedicina pauta-se pelo uso intensivo de conhecimentos da biologia molecular e da genômica, da bioinformática e, mais recentemente, das ferramentas de data science e de aprendizado de máquina. Tais abordagens são capazes de produzir diagnósticos mais precisos de determinadas doenças genéticas (ou a propensão de desenvolvê-las), novos insumos e medicamentos, e sistemas de monitoramento e gestão de dados ambientais e de estilo de vida para prevenir, monitorar ou tratar pacientes 1,2 .

Essa área registrou uma evolução significativa no volume de pesquisadores e empresas envolvidas, disponibilidade de recursos financeiros, número de trabalhos publicados e lançamento de novos produtos inovadores, principalmente nos Estados Unidos e na Europa. Com uma indústria de biotecnologia madura e uma população de média e alta rendas, cientistas dessas regiões promoveram diversos projetos nacionais de desenvolvimento científico e tecnológico nessa direção, conferindo dinamismo à pesquisa e aos negócios nesse setor. Para ilustrar o impacto de iniciativas nesse setor, entre 2014 e 2017 foram lançados nos Estados Unidos cerca de 75 mil testes genéticos no período, com uma entrada de, em média, dez novos testes por dia no mercado americano ${ }^{3}$.

A contribuição da medicina de precisão para a saúde pública, no entanto, tem sido alvo de questionamento 4 reiterado no atual contexto da pandemia de COVID-19, em que fica evidente o seu maior impacto sobre as populações vulneráveis em contexto de desigualdade social 5,6. Pesquisadores questionam o volume de recursos gastos no desenvolvimento da medicina de precisão quando comparados aos recursos para as ações tradicionais de saúde pública, e qual a real contribuição da medicina de precisão para o enfrentamento dos graves problemas de saúde globais 1,7. Também questiona-se como essa infraestrutura montada para atender uma elevada demanda por saúde suplementar pode ser usada para aprimorar os sistemas de saúde no combate aos efeitos negativos da pandemia, assim como direcionar a implementação de agendas de pesquisa em saúde pública mais equitativas e globalmente integradas ${ }^{8}$.

A crise sanitária sem precedentes causada pela epidemia de COVID-19 está impactando a pesquisa em todas as áreas do conhecimento e provocando uma reorientação das agendas de investigação. Revistas científicas criaram fast tracks para a rápida divulgação de artigos sobre a COVID-19 e editoriais foram escritos tecendo reflexões sobre o contexto, apontando prioridades para a pesquisa e como suas respectivas áreas poderão ser transformadas pela pandemia.

Nosso objetivo neste artigo é compreender como a comunidade internacional da medicina de precisão está reagindo à pandemia da COVID-19 e em que estão baseadas as suas abordagens e potenciais soluções sugeridas para a mitigação dos efeitos negativos causados pelo aumento das infecções pelo novo coronavírus. Para tanto, analisamos as narrativas difundidas por esses profissionais em artigos científicos, editoriais dos principais periódicos e instituições de pesquisa, e reflexão da medicina de precisão e outras publicações acadêmicas de grande circulação, no sentido de compreender como esses grupos têm produzido uma nova configuração sociotécnica para estabelecer suas contribuições no enfrentamento da pandemia e de seus efeitos.

\section{Metodologia}

A produção de publicações científicas faz parte da rotina do pesquisador profissional. Além de cumprirem o papel de divulgar o conhecimento produzido entre os pares e, assim, encontrar formas de se avançar na fronteira do conhecimento, tais documentos também são importantes referenciais para entender "como pensam" os cientistas, i.e. quais visões de mundo compartilham, como imaginam o futuro, quais pressupostos sustentam suas ideias, práticas e promessas e, também, como tais grupos buscam sensibilizar os formuladores de política e a opinião pública 9,10,11. Portanto, um exame sociológico do conteúdo de publicações científicas pode contribuir para o entendimento da atuação da comunidade científica para além de sua dimensão técnico-epistemológica, em que é possível identi- 
ficar posicionamentos de cunho cultural e político na forma como os grupos acadêmicos projetam e tentam legitimar suas agendas de pesquisa para a sociedade em geral 12.

Este artigo é resultado de pesquisa exploratória empírica baseada em combinação de métodos, constituído por: revisão da literatura de Sociologia do Conhecimento e da Saúde Coletiva; pesquisa documental (principalmente em artigos de opinião, perspectivas e comentários publicados entre fevereiro e setembro de 2020) e observação não participante em discussão virtual sobre o tema da medicina de precisão no contexto da COVID-19 em evento promovido por associação internacional de pesquisadores, policy-makers e da indústria, ocorrido em 03 de setembro de 2020, que contou com a participação dos autores deste trabalho como ouvintes. Assim, reservou-se espaço para a análise de editoriais acadêmicos, comentários, perspectivas e notícias publicadas em sítios web selecionados.

Entre os meses de fevereiro e setembro de 2020 foi feita uma busca na base de artigos do PubMed, em que foram selecionados 28 documentos oriundos de 18 fontes: seis artigos originais, dez artigos de comentário, cinco perspectivas, um editorial acadêmico e seis notícias publicadas em organismos internacionais, associações, jornais ou boletins de notícia relevantes. Dada a grande quantidade de artigos científicos publicados sobre a COVID-19, a opção foi centralizar a busca em revistas dessa comunidade de pesquisa em particular, sem incorrer no risco de analisar editoriais de áreas mais tradicionais das ciências que se interessam pelo tema da medicina de precisão, mas que conferem outra abordagem que não nos interessa aqui. É importante ressaltar também que não estamos considerando aqui a produção científica relacionada à biologia molecular de patógenos por considerar que extrapolam os limites da medicina de precisão.

Por isso, foi dada especial atenção aos artigos publicados nos seis periódicos: Journal of Precision Medicine; Personalized Medicine/Future Medicine; Journal of Personalised Medicine; Precision Medicine Sciences; Public Health Genomics; e Precision Clinical Medicine. Documentos relevantes também foram encontrados nos sítios da Divisão de Genômica e Saúde de Precisão do Centro de Controle e Prevenção de Doenças (CDC) dos Estados Unidos; de associações internacionais relevantes como a Coalizão de Medicina Personalizada (PMC, em inglês), Consórcio Internacional para Medicina Personalizada (ICPerMed, em inglês), Oncologia Alvo e Saúde IT Analítica, e de organismos internacionais, como a Organização Mundial da Saúde (OMS), Health Europa e do Fórum Econômico Mundial. Por fim, selecionamos o evento online COVID-19 and Personalized Medicine: Current Status and Lessons Learned promovido pela PMC para examinar as narrativas e discussões promovidas entre os participantes, de maneira a identificar importantes percepções sobre como a comunidade tem respondido aos desafios impostos pela pandemia, e como eles enxergam o futuro desta relação. Detalhes sobre os documentos analisados na pesquisa documental estão disponíveis no Quadro 1.

\section{Resultados e discussão}

Dentre os aspectos mais gerais evidenciados pela pesquisa documental, chamou atenção o quase silêncio das revistas específicas de medicina de precisão sobre o tema da COVID-19 até meados de junho de 2020. Apenas um comentário na revista Journal of Precision Medicine abordou a pandemia de maneira direta no número publicado em março. Já na edição de junho, a revista publicou quatro artigos sobre a COVID-19 e uma versão estendida do comentário publicado em março.

A pesquisa documental feita entre os meses de abril e setembro de 2020 mostrou que parte importante das reivindicações feitas pelos participantes da comunidade internacional em medicina de precisão faz referência à pandemia da COVID-19 como um contexto de oportunidade, seja para o desenvolvimento de novos produtos e serviços médicos para potenciais novos clientes, seja para uma aceleração no afrouxamento regulatório para demandas tradicionais deste setor, como maior rapidez na aprovação de produtos por parte das agências reguladoras, maior integração entre os setores público e privado para a viabilização de soluções em saúde pública, regras mais flexíveis para o acesso e compartilhamento de dados biológicos de pacientes e, também, uma reforma no aparato regulatório existente, para dinamizar a comercialização de testes moleculares e dispositivos médicos em larga escala.

Para interpretar esses achados, destacamos a contribuição dada por, pelo menos, três eixos de pesquisa fundamentais sobre a dinâmica do conhecimento e da inovação em saúde contemporânea, 


\section{Quadro 1}

Detalhes do material consultado na pesquisa documental $(n=28)$.

\begin{tabular}{|c|c|c|c|c|}
\hline Periódico & Seção & Título & Autores & Data (2020) \\
\hline \multirow[t]{7}{*}{ Journal of Precision Medicine } & \multirow[t]{2}{*}{ Comentário } & \multirow{2}{*}{$\begin{array}{c}\text { Personalized Medicine in the Aftermath of } \\
\text { COVID-19 (comentário de abril estendido } \\
\text { e republicado) }\end{array}$} & \multirow[t]{2}{*}{ Abrahams 13} & Março \\
\hline & & & & Junho \\
\hline & \multirow[t]{5}{*}{ Artigo } & $\begin{array}{c}\text { Diagnosing and Tracking COVID-19 } \\
\text { Infections Leveraging Next-Gen. Sequencing }\end{array}$ & Scherer \& Scherer 33 & Junho \\
\hline & & $\begin{array}{l}\text { Leveraging Real-World Data for COVID-19 } \\
\text { Research: Challenges and Opportunities }\end{array}$ & Reynolds et al. 34 & \multirow[t]{4}{*}{ Junho } \\
\hline & & $\begin{array}{c}\text { COVID-19 and Pharmacogenomics: An } \\
\text { Association that Warrants Comprehensive } \\
\text { Assessment }\end{array}$ & Kekic \& Asudani 35 & \\
\hline & & The Journey to a COVID-19 Diagnostic Test & Loewy 36 & \\
\hline & & $\begin{array}{l}\text { How Cancer Patients Should Respond to the } \\
\text { COVID-19 Pandemic }\end{array}$ & Power 37 & \\
\hline Precision Medicine Sciences & Editorial & $\begin{array}{l}\text { Retrospect and Prospect of Precision } \\
\text { Medical Sciences }\end{array}$ & Feng 38 & 18 de junho \\
\hline Personalized Medicine/Future Medicine & - & - & - & - \\
\hline Journal of Personalized Medicine & Artigo & $\begin{array}{l}\text { Postulated Adjuvant Therapeutic Strategies } \\
\qquad \text { for COVID-19 }\end{array}$ & Ferreira et al. 39 & 5 de agosto \\
\hline Public Health Genomics & - & - & - & - \\
\hline \multirow[t]{4}{*}{ Precision Clinical Medicine } & \multirow[t]{4}{*}{ Perspectiva } & $\begin{array}{c}\text { A Precision Medicine Approach to Managing } \\
2019 \text { Novel Coronavirus Pneumonia }\end{array}$ & Wang et al. 40 & 4 de fevereiro \\
\hline & & $\begin{array}{l}\text { Sustained Research Fund and Dedicated } \\
\text { Research Center to Prepare for the Next } \\
\text { Pandemic }\end{array}$ & Jia \& Wu 41 & 11 de abril \\
\hline & & $\begin{array}{l}\text { Host/Genetic Factors Associated with } \\
\text { COVID-19 Call for Precision Medicine }\end{array}$ & Thierry 42 & 21 de julho \\
\hline & & $\begin{array}{l}\text { Calling for a United Action to Defeat } \\
\text { COVID-19 }\end{array}$ & Overby et al. 19 & 6 de agosto \\
\hline
\end{tabular}

(continua) 
Quadro 1 (continuação)

\begin{tabular}{|c|c|c|c|c|}
\hline Associações & Seção & Título & Autores & Data (2020) \\
\hline \multirow[t]{5}{*}{$\begin{array}{l}\text { CDC Genomics \& Precision Health } \\
\text { Blog }\end{array}$} & \multirow[t]{7}{*}{ Comentário } & $\begin{array}{c}\text { From Precision Medicine to Precision Public } \\
\text { Health: Beyond the Pandemic }\end{array}$ & Khoury 43 & 19 de junho \\
\hline & & $\begin{array}{l}\text { Toward More Precision in Implementation } \\
\text { Science in the Age of COVID-19 }\end{array}$ & Clyne et al. 44 & 26 de junho \\
\hline & & $\begin{array}{c}\text { The Public Health Impact of COVID-19: Why } \\
\text { Host Genomics? }\end{array}$ & Khoury et al. 45 & 21 de abril \\
\hline & & $\begin{array}{l}\text { Using Digital Technologies in Precision } \\
\text { Public Health: COVID-19 and Beyond }\end{array}$ & Khoury et al. 46 & 6 de abril \\
\hline & & $\begin{array}{c}\text { Precision Public Health and the COVID-19 } \\
\text { Response }\end{array}$ & Rasmussen et al. 16 & 17 de agosto \\
\hline $\begin{array}{l}\text { International Consortium for } \\
\text { Personalised Medicine }\end{array}$ & & $\begin{array}{l}\text { How Personalised Medicine will Transform } \\
\text { Healthcare by 2030: The ICPerMed Vision }\end{array}$ & Vicente et al. 47 & 28 de abril \\
\hline Target Oncology & & $\begin{array}{c}\text { COVID-19 Presents an Opportunity for } \\
\text { Precision Medicine to Play Expanded Role } \\
\text { in Care }\end{array}$ & Patel et al. 48 & 22 de maio \\
\hline Health IT Analytics & Notícias & $\begin{array}{l}\text { "All of Us" Precision Medicine Program to } \\
\text { Collect COVID-19 Data }\end{array}$ & Kent 49 & 17 de junho \\
\hline Organismos internacionais & Seção & Título & Autores & Data (2020) \\
\hline Organização Mundial da Saúde & Perspectiva & $\begin{array}{c}\text { Precision Medicine for COVID-19: A Call for } \\
\text { Better Clinical Trials }\end{array}$ & Shrestha 50 & 2 de junho \\
\hline Health Europa & \multirow[t]{2}{*}{ Notícias } & $\begin{array}{c}\text { Al Precision Medicine Mining Finds } 13 \\
\text { Human COVID-19 Risk Genes }\end{array}$ & Health Europa 51 & 8 de maio \\
\hline Forum Econômico Mundial & & $\begin{array}{c}\text { Global Leaders Unlock Ways for Precision } \\
\text { Medicine to Fight COVID-19, Cancers and } \\
\text { Emerging Infectious Diseases }\end{array}$ & World Economic Forum 52 & 27 de maio \\
\hline Evento & Seção & Título & Autores & Data (2020) \\
\hline $\begin{array}{l}\text { PMC COVID-19 and Personalized } \\
\text { Medicine }\end{array}$ & - & $\begin{array}{l}\text { COVID-19 and Personalized Medicine: } \\
\text { Current Status and Lessons Learned }\end{array}$ & $\begin{array}{l}\text { Personalized Medicine } \\
\text { Coalition } 53\end{array}$ & 3 de setembro \\
\hline Jornais/Boletim de notícias & Seção & Título & Autores & Data (2020) \\
\hline Blogs Scientific American & Comentário & $\begin{array}{c}\text { The Inflated Promise of Genomic Medicine. } \\
\text { COVID-19 has Laid Bare the Need to } \\
\text { Reconsider the Hope and Money we Invest in } \\
\text { Genetics Research }\end{array}$ & Parens 6 & 1 de junho \\
\hline The New York Times & \multirow[t]{3}{*}{ Notícias } & $\begin{array}{l}\text { DNA Inherited from Neanderthals May } \\
\text { Increase Risk of COVID-19: The Stretch of Six } \\
\text { Genes Seems to Increase the Risk of Severe } \\
\text { IIIness from the Coronavirus }\end{array}$ & Zimmer 54 & 4 de julho \\
\hline The Wall Street Journal & & $\begin{array}{c}\text { COVID-19 Raises Questions About the Value } \\
\text { of Personalized Medicine }\end{array}$ & Marcus 5 & 8 de maio \\
\hline Jornal da USP & & $\begin{array}{c}\text { Estudo Genético Pretende Mapear Grupos } \\
\text { de Risco da COVID-19 }\end{array}$ & 55 & Maio \\
\hline
\end{tabular}


referentes (1) aos estudos sobre o processo de biomedicalização das sociedades; (2) às transformações nas políticas do welfare state e aos novos modelos de gestão adotados pelos sistemas de saúde; e (3) ao ininterrupto processo de transnacionalização dos regimes de produção do conhecimento biomédico, que excluiu a maior parte dos países subdesenvolvidos e reservou a estes o papel de consumidores de tecnologias em saúde.

Esses três eixos situam a experiência da medicina de precisão em tempos de pandemia como um fenômeno sociotécnico sui generis que opera e se transforma dentro de uma dinâmica cultural, política e tecnológica mais ampla. Identificamos pelo menos quatro processos sociológicos centrais evidenciados pela pesquisa documental. São eles: a presença de um movimento pró-flexibilização do aparato regulatório para acesso e compartilhamento de dados genéticos e biológicos de pacientes, fundamental para dar maior dinamismo para o setor de pesquisa e dos negócios em medicina de precisão; a difusão do conceito de precision public health como ferramenta de Bandwagon (i.e., nesse contexto, conceito idealizado para servir como ferramenta de promoção política da comunidade científica e sua tradução, em espaços sociais mais amplos) relevante para integrar medicina de precisão e o sistema de saúde e, finalmente, o papel dos sistemas de saúde como parceiros em projetos com o setor privado, mas compradores de seus resultados.

\section{Ênfase na busca de uma solução tecnológica para a pandemia}

Um ponto enfatizado na literatura é a importância de aproveitar o contexto de oportunidade valorizando a possível contribuição tecnológica da medicina de precisão para o enfrentamento da COVID-19. No comentário publicado na Journal of Precision Medicine, Abrahams 13 afirma que os efeitos variáveis do vírus em diferentes indivíduos tornam imperativo o desenvolvimento e a implantação de instrumentos diagnósticos que possam orientar estratégias de prevenção focadas na população em maior risco. Esses instrumentos poderiam contribuir para a formação de sistemas sentinelas de vigilância e monitoramento de casos em tempo real. O autor enfatiza, portanto, a necessidade de pesquisas sobre os fatores genéticos que contribuem para a progressão diferenciada da doença em cada paciente.

Nos documentos analisados, um mote central proposto pela medicina de precisão é a possibilidade de identificar genes ou variantes genéticas que possam explicar as diferenças na evolução da doença e identificar grupos em alto risco. Alguns projetos de pesquisa têm sido realizados com esses objetivos. No Reino Unido, o projeto da Genomics England em parceria com o GenOMICC Consortium, liderado pela Universidade de Edimburgo, Escócia, está analisando o sequenciamento do genoma de 20 mil pessoas que foram severamente afetadas pela COVID-19, que devem ser comparados com genomas de pessoas que foram afetadas de forma leve, sem a necessidade de internação hospitalar. No Brasil, na mesma direção, um estudo realizado na Universidade de São Paulo visa a sequenciar o genoma de pessoas que tiveram quadros leve e grave de COVID-19, e identificar grupos genéticos associados aos dois desfechos.

A ênfase nas oportunidades tecnológicas esteve presente no evento analisado, que compõe o quadro de fontes da pesquisa documental. Ocorrida em 3 de setembro de 2020, a conferência virtual promovida pela PMC debateu, na maior parte do tempo, as iniciativas de combate à pandemia principalmente no âmbito das políticas públicas de nível federal, no caso dos Estados Unidos, e nacionais, na Europa. Uma discussão específica sobre a medicina de precisão e suas contribuições na pandemia ocorreu nos trinta minutos finais do evento virtual. Chamou a atenção a maneira como os especialistas abordaram aquelas que seriam possíveis respostas para os desafios da pandemia.

Para o pesquisador Toni Andreu (Diretor Científico da Infraestrutura Europeia para Medicina Translacional - EATRIS), uma vez que está claro que a COVID-19 acomete pessoas de diferentes idades e de maneiras distintas, isto pode ser um gatilho para o estudo dos aspectos genéticos e moleculares dos pacientes. Se ainda não se sabe qual é o padrão que agrava a saúde das pessoas que contraem o novo coronavírus, então isto pode ser entendido como potencial pergunta de pesquisa a ser perseguida pela medicina de precisão.

Para o interlocutor, "nós temos a oportunidade de ser mais agressivos e diretos na promoção dessas abordagens, partindo de, mas indo além, da medicina genômica". Para o pesquisador, essa é uma "chance para impulsionar a indústria de biomarcadores, assim como de angariar mais recursos e instrumentos políticos para promover áreas com potencial de futuro como a comercialização de testes de Neuroprofilling direto ao consu- 
midor etc.". Nessa direção, o pesquisador Dr. Jay Wohlgemuth (Vice-Presidente de Ciência e Inovação da Quest Diagnostics) faz um questionamento retórico aos outros participantes: "As pessoas precisam fazer o teste em suas casas: se elas vão fazer o teste para COVID-19, por que não fazer o teste para câncer de cólon, câncer de mama, doença de Alzheimer, etc.?”. Essa manifestação aponta que existe uma lógica de mercado importante conduzindo as ações das associações da medicina de precisão durante a pandemia, que tem no setor privado um importante financiador e promotor de novas tecnologias.

A justificativa de que os grandes laboratórios não estão conseguindo entregar os testes com a eficiência esperada foi usada como argumento para fortalecer a cadeia de produtores de testes durante a pandemia. Para o pesquisador Dr. Michael Sherman (Co-Chair do Comitê de Política Pública da PMC e Vice-Presidente Sênior do Direção Médica da Harvard Pilgrim Healthcare), "é visível que a oferta de testes é um grande gargalo causado pela incapacidade dos grandes laboratórios de prover esses produtos em quantidade necessária, então a sociedade deve se movimentar para suprir essa demanda".

A preocupação com fatores sociais, quando presente, orienta-se pelo discurso de garantir o acesso da população às novas tecnologias de precisão por meio de políticas de regulação e reembolso. Em geral, tanto nos artigos revisados quanto no evento observado não há referência ao enfrentamento das desigualdades sociais associadas à maior vulnerabilidade à COVID- 19 .

\section{Acesso e compartilhamento de dados}

Acessar e compartilhar dados biomédicos da população é um assunto presente nos editoriais e eventos da medicina de precisão. Esse é um debate em expansão no campo da bioética e suas implicações no campo da medicina de precisão têm sido estudadas por grupos de pesquisa na Europa e nos Estados Unidos 14. A produção de um novo arcabouço regulatório faz parte da agenda política da medicina de precisão, uma vez que os stakeholders buscam continuamente viabilizar o acesso mais eficiente e o compartilhamento de dados juridicamente assegurado, algo que interessa aos cientistas e empreendedores. Conforme apontam Blasimme et al. 15, ao longo dos últimos anos aspectos como a autonomia na gestão dos dados e a garantia da privacidade das informações ganharam ampla atenção nesse setor. Para os autores, apesar dessas preocupações aparecerem nos guias de diversas organizações estudadas, isto não ocorreu com o mesmo dinamismo para temas como a interoperabilidade e accountability na governança dos dados, o que mostra que ainda não há um sistema coeso de políticas e incentivos capaz de conciliar os interesses e as expectativas dos parceiros deste setor.

O tema da governança dos dados ressurgiu como uma agenda importante na pandemia da COVID-19. De acordo com três publicações selecionadas, cientistas, agências de governo e associações da medicina de precisão entendem esse momento como uma excelente oportunidade de mostrar para a sociedade a importância de se flexibilizar não só a rigidez com que são feitos os ensaios clínicos de novas drogas, mas também o acesso e compartilhamento de dados genéticos de pacientes. Segundo propositores da precision public health, os sistemas de saúde deveriam ser parceiros mais atuantes nesse processo, já que isso viabilizaria o acesso a diagnósticos mais precisos como estratégia mais efetiva de rastreamento, monitoramento e controle da pandemia 16,17 .

O diálogo entre medicina de precisão e Saúde Pública, presente como um aspecto fundamental encontrado nos documentos analisados, pode evidenciar uma mudança importante na maneira como os sistemas de saúde têm buscado alternativas para o processo de subfinanciamento de seus projetos: reflexo da redução da participação do Estado na governança das políticas públicas de saúde e, também, traço marcante do processo de biomedicalização 18 , em que uma característica relevante refere-se à vigilância da saúde ser cada vez mais uma responsabilidade individual e não coletiva.

Por outro lado, esse maior diálogo deixa explícita uma mudança na forma como o conhecimento biomédico contemporâneo tem sido produzido, distribuído e consumido, e como os novos acontecimentos impactaram na maneira como se dá a sua governança. Isso pode dar mais pluralidade aos regimes de produção de conhecimento em saúde, que não fiquem apenas circunscritos à burocracia do Estado. 


\section{Precision public health}

Os documentos analisados neste artigo mostram que a pandemia da COVID-19 colocou em xeque o uso do conceito de medicina de precisão como relevante para o campo da Saúde Pública 19. No entanto, ajudou a fortalecer um movimento para a difusão do conceito de precision public health. O termo apareceu com destaque nos editoriais, comentários e notícias publicados a partir de junho de 2020, sobretudo em publicações do CDC dos Estados Unidos. A divulgação desse termo chama atenção por se tratar de um importante recurso discursivo utilizado pelos pesquisadores em sua agenda de Bandwagon científico pós-pandemia.

$\mathrm{O}$ conceito de precision public health surgiu antes da pandemia, e veio substituir o antigo conceito de public health genomics de forma similar ao deslocamento semântico que ocorreu na substituição da medicina personalizada para a medicina de precisão. Os defensores da precision public health argumentam que a denominação anterior dá demasiada ênfase à genômica, mas que hoje transcende seus limites incorporando outros biomarcadores, assim como dados populacionais relacionados aos indivíduos 20 .

O termo vem sendo usado por importantes pesquisadores a partir de 2017 para fomentar a ideia de que a integração entre lógicas institucionais da saúde pública e da medicina de precisão é um exercício possível e necessário 21. Nossa abordagem, no entanto, entende esse como um importante recurso político da comunidade de pesquisa para ampliar sua esfera de influência no debate entre tecnologia e Saúde Pública.

Para Soraya de Chadarevian 22 (p. 206) "Nomes importam. Eles não são apenas rótulos ou termos de referência para relatos históricos, mas ferramentas estratégicas". Powell et al. 23 também discutem a prática de dar nomes a campos científicos, e traçam esse paralelo sobre o papel político dessas nomenclaturas. Nesse sentido, precision public health propicia um novo início para a ideia de uma saúde pública voltada para indivíduos ou grupos específicos da população, sem trazer o desgaste das promessas não cumpridas da public health genomics.

A difusão do termo precision public health funciona como uma importante ferramenta de Bandwagon científico na medicina de precisão, conforme discussão apresentada por Au \& Silva 2 em um estudo sobre este fenômeno na comunidade de pesquisa em medicina de precisão na China e no Brasil, interpretando a medicina de precisão como um conceito integrador de áreas de conhecimento, abordagens médicas, setores da indústria da saúde e atores da sociedade civil interessados em fazer avançar a pesquisa e o desenvolvimento, e os negócios em saúde personalizada. Nesse sentido, estratégias de Bandwagon científico são fundamentais para viabilizar um processo de institutional building entre atores que possam interferir nessa agenda, assim como para que o movimento ganhe amplitude não apenas no eixo técnico-acadêmico, mas também no político-econômico.

$\mathrm{O}$ interesse na difusão do termo precision public health se apresentou como um aspecto importante também em comentários recentes em agências sanitárias, em boletins de organismos internacionais como a OMS, jornais de notícias e até no evento da PMC ocorrido em 03 de setembro de 2020, em que ficou evidente uma agenda de aproximação da medicina de precisão como provedora de soluções em saúde pública. No contexto da pandemia de COVID-19, stakeholders da medicina de precisão buscam mostrar que a abordagem pode contribuir para a saúde das populações. Mas não fica claro, ainda, se essa aproximação visa a compartilhar ações na governança da produção de novos conhecimentos e tecnologias que fiquem acessíveis aos usuários do sistema de saúde como um todo, ou se as parcerias com os sistemas de saúde visam a acessar material genético e biológico em larga escala para viabilizar projetos cujo resultado será mais caro e, portanto, inacessível.

\section{Conhecimento biomédico não é global}

Em grande medida, a análise do editorial e dos comentários indica que os entusiastas da medicina de precisão buscam continuamente passar a ideia de que a pandemia será vencida com uma integração mundial de conhecimento e de ações de governo. No entanto, os resultados dessa crise sanitária sem precedentes têm mostrado que a relação entre os países é assimétrica em termos das capacidades de pesquisa locais. 
Os editoriais têm sido atuantes para fortalecer o mito do conhecimento biomédico como uma entidade global, sugerindo que todos participamos da produção de inovações neste setor e que nos beneficiamos diretamente delas. No entanto, a pandemia deixou ainda mais claro que, no mundo, existem os que produzem e os que consomem conhecimento biomédico e em saúde, e que isto tem grande impacto na contenção de crises sanitárias.

Lock \& Nguyen 24 constatam que, desde o fim dos anos 1980, a comunidade científica internacional tem empreendido um grande esforço de padronização das políticas nacionais de ciência, tecnologia e inovação em saúde, em que regimes locais de produção do conhecimento biomédico foram transformados significativamente ao longo das últimas décadas.

Entretanto, como argumentam Costa \& Silva 25 , nos países em desenvolvimento essa mudança foi verificada pelo aumento nos custos de aquisição, gestão e manutenção de equipamentos, insumos de laboratório, sequenciadores etc. que encareceu a pesquisa. Isso revelou desigualdades em que populações de baixa renda não têm acesso a tais inovações e tecnologias 26 . Em um contexto no qual o cuidado à saúde é cada vez mais terceirizado para tecnologias, empreendedores e comunidades locais, ocorrendo fora do encontro clínico médico/paciente 27, a pandemia da COVID-19 mostrou a fragilidade da medicina de precisão como abordagem para a Saúde Pública.

\section{Sistemas de saúde como parceiros e clientes}

Como vimos na análise do material levantado pela pesquisa documental, o afrouxamento do aparato regulatório para a comercialização de novos produtos é a principal demanda da comunidade de medicina de precisão. Desde antes da pandemia, como mostra Stuart Hogarth 28, idealizadores dos negócios de testes genéticos e medical devices na Europa e nos Estados Unidos sustentam que é necessária uma mudança no quadro das políticas de regulação para garantir a venda direta para o consumidor.

Esse argumento é retomado nos editoriais e comentários. A conferência da PMC levantou um debate importante sobre a condução das medidas de saúde pública globais, e os pesquisadores europeus fizeram questionamentos sobre as medidas adotadas nos Estados Unidos. No que diz respeito aos novos consórcios internacionais formados para viabilizar a produção de vacinas, houve um debate sobre os Estados Unidos optarem por ficar de fora das redes de colaboração em saúde pública por razões políticas. Há uma postura muito distinta entre os especialistas americanos e os europeus em relação a isso, tópico que merece um estudo mais cuidadoso.

Essa discussão mostra que existe um conflito entre o que podemos chamar de duas "geopolíticas" que coexistem no setor de saúde nos Estados Unidos e na Europa: uma relacionada à tradição em serviços, pesquisa e produção de tecnologias em saúde pública, que tem base sólida principalmente nas instituições de saúde europeias; e outra vinculada ao dinamismo dos mercados e dos negócios em medicina de precisão, que embora tenha uma agenda de advocacy tanto nos Estados Unidos quanto em países europeus, mantém-se como uma lógica dominante em volume de investimentos e de mercado no país norte-americano. Ambas as vertentes têm ganhado espaço durante a pandemia, seja com o discurso da promoção de tecnologias para a COVID-19 como bens públicos transnacionais 8 , seja por meio da bandeira de afrouxamento do aparato regulatório para as novas tecnologias em saúde.

\section{Conclusões}

Dada a projeção dessa comunidade científica e o grande volume de recursos que ela tem recebido ao longo dos últimos anos, pode-se admitir que, pelo menos até o momento em que este artigo foi produzido, as agendas propostas pela medicina de precisão para combate à pandemia têm se mostrado tímidas e pouco ambiciosas. Entende-se que uma agenda adequada e eficaz, empreendida por essa comunidade, poderia ser articulada entre os seus diversos eixos de atuação na esfera acadêmica, na indústria da saúde e na promoção da saúde pública; muito embora o foco destes atores seja a proposição de soluções no plano individual/personalizado, esses grupos têm um estoque de conhecimento, de infraestrutura científica e de influência política que poderia propor intercâmbios mais expressivos com as políticas científicas e tecnológicas em saúde, com a saúde coletiva e, também, com abordagens de amplo alcance da saúde global. 
A despeito disso, verificou-se nos depoimentos em editoriais e no evento online um direcionamento para a prospecção de potenciais novos negócios, flexibilização de mecanismos de regulação e, na esfera acadêmica, uma contínua formulação de questões/perguntas de pesquisa, inovações metodológicas para a prática da pesquisa em laboratório e desenho de novas hipóteses. Isso é, sem dúvida, importante e necessário, mas em contextos de emergência global, uma atuação política mais propositiva poderia encontrar atalhos no desenvolvimento de tecnologias que pudessem conter, ou mesmo desacelerar, o crescimento das infecções.

Refletir sobre as causas dessa aparente desarticulação é uma tarefa mais trabalhosa, que demandaria coleta de dados mais específica. Entretanto, entende-se que esse fenômeno pode ser explicado em parte pelo desmonte das políticas do Welfare State no Ocidente ao longo das últimas três décadas, assim como pela maior intersecção entre lógicas institucionais dos setores público e privado. Kavanagh \& Singh 29 argumentam que a pandemia deixou explícita a redução da capacidade política do Estado contemporâneo em coordenar esses processos e que, ainda que fosse competente nessa atividade, não reuniria todas as competências necessárias; a economia política da pandemia da COVID-19 articula novos atores, processos e desdobramentos que vão além da alçada da administração estatal.

Também se apresenta como um achado importante o papel da pandemia como oportunidade para a comercialização de novos produtos direto ao consumidor, difundido por parte dos atores que compuseram a discussão em evento da PMC em setembro de 2020. Ficou evidente que entidades do setor privado têm promovido ativamente o discurso para aproveitar a pandemia a fim de flexibilizar o longo processo de aprovação e registro de novas tecnologias da medicina de precisão.

Outro aspecto relevante a ser mencionado diz respeito ao interesse de alguns editoriais e comentários no fortalecimento do conceito de precision public health. A defesa desse termo é anterior à pandemia 21, no entanto, a noção de uma saúde pública de precisão ganhou destaque nos editoriais da revista Precision Clinical Medicine e na Divisão de Genômica e Saúde de Precisão do CDC. Esse é um ponto relevante dado que uma das grandes críticas que são feitas à medicina de precisão se centra na sua modesta contribuição para a saúde pública.

A pesquisa documental sugere que a medicina de precisão enfrenta desafios reais para se projetar para além de suas promessas tecnológicas. Como argumenta Parthasarathy ${ }^{30}$, as tecnologias por si só não resolvem os reais problemas agravantes da pandemia, que são primordialmente de ordem socioeconômica, e dispor apenas desta via pode explicar a pouca eficácia de medidas sanitárias adotadas durante a pandemia.

A medicina de precisão se apresenta ora como alternativa de melhores e mais precisas tecnologias, ora como soluções para a prevenção de doenças no plano personalizado, mas falha na integração mais equânime entre acesso e promoção de saúde pública. Como sustentam Ventura et al. 31 (p. 2): "fica evidente que a resposta às emergências só é possível na presença de sistemas de saúde bem estruturados, com capacidade de vigilância e de pesquisa, conduzidos por uma massa crítica de profissionais bem formados e capacitados para ação nestas três vertentes".

As respostas à COVID-19 que se mostraram mais eficazes até aqui fizeram uso de tecnologias já difundidas e com trajetória conhecida em epidemias anteriores 32, mostrando que é fundamental resgatar o aprendizado em saúde pública para que as tecnologias sejam encaixadas em sistemas sociotécnicos complexos e politicamente mais viáveis. A análise feita aqui mostra os limites da abordagem da medicina de precisão, que embora seja bastante eficiente no acesso a recursos para grandes (e caros) projetos, esbarra em barreiras importantes por não serem capazes de capturar os desafios sociais presentes na agenda de promoção de saúde pública. 


\section{Colaboradores}

R. G. L. Silva participou da concepção e escrita do artigo, desenho da metodologia, revisão da literatura, organização da pesquisa documental, coleta, seleção e análise dos dados, revisão de todas as versões do manuscrito e sua submissão. J. A. B. Iriart participou da concepção e escrita do artigo, desenho da metodologia, revisão da literatura, coleta, seleção e análise de dados, e revisão de todas as versões do manuscrito.

\section{Informações adicionais}

ORCID: Renan Gonçalves Leonel da Silva (00000001-9679-6389); Jorge Alberto Bernstein Iriart (0000-0002-9518-1240).

\section{Agradecimentos}

Os autores agradecem ao Departamento de Medicina Preventiva, Faculdade de Medicina, Universidade de São Paulo e ao Instituto de Saúde Coletiva, Universidade Federal da Bahia, pelo apoio institucional fornecido para viabilizar a execução deste trabalho. Os mesmos agradecem também à Coordenação de Aperfeiçoamento de Pessoal de Nível Superior (CAPES) pela concessão de bolsa de pós-doutorado ao primeiro autor via INCT-IATS processo no 88887.373046/2019-00 e ao Conselho Nacional de Desenvolvimento Científico e Tecnológico (CNPq) pela bolsa de produtividade fornecida ao segundo autor. O primeiro autor agradece, também, o apoio institucional fornecido pelo Health Ethics and Policy Lab do Instituto Federal Suíço de Tecnologia de Zurique (ETH Zürich), que tornou possível a finalização da análise dos dados, submissão e revisão da versão final do manuscrito.

\section{Referências}

1. Iriart JAB. Medicina de precisão/medicina personalizada: análise crítica dos movimentos de transformação da biomedicina no início do século XXI. Cad Saúde Pública 2019; 35:e00153118.

2. Au L, Silva RGL. Globalizing the scientific bandwagon: trajectories of precision medicine in China and Brazil. Sci Technol Human Values 2020; 46:192-225.

3. Phillips KA, Deverka PA, Hooker GW, Douglas MP. Genetic test availability and spending: where are we now? Where are we going? Health Aff (Millwood) 2018; 37:710-6.

4. Joyner M, Paneth N. Seven questions for personalized medicine. JAMA 2015; 314:999-1000.

5. Marcus AD. COVID-19 raises questions about the value of personalized medicine. The Wall Street Journal 2020; 8 may. https://www. wsj.com/articles/covid-19-raises-questionsabout-the-value-of-personalized-medi cine-11588949927.

6. Parens E. The inflated promise of genomic medicine: COVID-19 has laid bare the need to reconsider the hope and money we invest in genetics research. https://blogs.scientifi camerican.com/observations/the-inflatedpromise-of-genomic-medicine (acessado 10/ Ago/2020).

7. Cooper R, Paneth N. Precision medicine: course correction urgently needed. https:// www.statnews.com/2020/03/03/precisionmedicine-course-correction-urgently-need ed/ (acessado em 10/Ago/2020).

8. Silva RGL. Uma reflexão sobre agendas de pesquisa e inovação em saúde na pandemia da COVID-19. Boletim DPCT COVID-19 2020; (14). https://www.unicamp.br/unicamp/coro navirus/uma-reflexao-sobre-agendas-de-pes quisa-e-inovacao-em-saude-na-pandemia-dacovid.

9. Knorr-Cetina K. Epistemic cultures: how sciences make knowledge. Cambridge: Harvard University Press; 1999.

10. Jasanoff S. States of knowledge: the co-production of science and the social order. London/New York: Routledge; 2004.

11. Clarke A, Fujimura J. The right tools for the job: at work in twentieth-century life sciences. Princeton: Princeton University Press; 2014.

12. Tutton R. Genomics and the reimagining of personalized medicine. Farnham: Ashgate Publishing; 2014.

13. Abrahams E. Personalized medicine in the aftermath of COVID-19. J Precis Med 2020; 1. https://www.thejournalofprecisionmedi cine.com/personalized-medicine-in-the-after math-of-covid-19/.

14. Blasimme A Vayena E. "Tailored-to you": public engagement and the political legitimation of precision medicine. Perspect Biol Med 2016; 59:172-88. 
15. Blasimme A, Fadda M, Schneider M, Vayena E. Data sharing for precision medicine: policy lessons and future directions. Health Aff (Millwood) 2018; 37:702-9.

16. Rasmussen SA, Khoury MJ, del Rio C. Precision public health as a key tool in the COVID-19 response. JAMA 2020; 324:933-4.

17. Clyne M, Chambers DA, Khoury MJ. Toward more precision in implementation science in the age of COVID-19. https://blogs.cdc.gov/ genomics/2020/06/26/toward-more-preci sion/ (acessado em 29/Jul/2020).

18. Clarke AE, Shim JK, Mamo L, Fosket JR, Fishman JR. Biomedicalization: technoscientific transformations of health, illness, and U.S. biomedicine. Am Sociol Rev 2003; 68:161-94.

19. Overby M, Pu Q, Wei X, Wu M. Calling for a united action to defeat COVID-19. Precis Clin Med 2020; 3:235-9.

20. Metzler I. Biomarkers and their consequences for the biomedical profession: a social science perspective. Per Med 2010; 7:407-20.

21. Khoury MJ, Bowen MS, Clyne M, Dotson WD, Gwinn ML, Green RF, et al. From public health genomics to precision public health: a 20-year journey. Genet Med 2018; 20:574-82.

22. Chadarevian S. Designs for life: molecular biology after World War II. Cambridge: Cambridge University Press; 2002.

23. Powell A, O’Malley MA, Müller-Wille S, Calvert J, Dupré J. Disciplinary baptisms: a comparison of the naming stories of genetics, molecular biology, genomics, and systems biology. Hist Philos Life Sci 2007; 29:5-32.

24. Lock M, Nguyen V-K. An anthropology of biomedicine. Oxford: Wiley-Blackwell; 2010.

25. Costa M, Silva RGL. A dinâmica do conhecimento biomédico e em saúde: uma interpretação sociológica. Sociologias 2019; 21:18-47.

26. Hilgartner S, Gibbon S, Prainsack B, Lamoreaux J. Handbook of genomics, health and society. 2nd Ed. New York: Routledge International Handbooks; 2018.

27. Biehl J, Moran-Thomas A. Symptom: subjectivities, social ills, technologies. Annu Rev Anthropol 2009; 38:267-88.

28. Hogarth S, Javitt G, Melzer D. The current landscape for direct-to-consumer genetic testing: legal, ethical, and policy issues. Annu Rev Genomics Hum Genet 2008; 9:161-82.

29. Kavanagh MM, Singh R. Democracy, capacity, and coercion in pandemic response: COVID-19 in comparative political perspective. J Health Polit Policy Law 2020; 45:997-1012.

30. Parthasarathy S. More testing alone will not get us out of this pandemic. Nature 2020; 585:8.

31. Ventura DFL, Ribeiro H, Giulio GM, Jaime PC, Nunes J, Bógus CM, et al. Desafios da pandemia de COVID-19: por uma agenda brasileira de pesquisa em saúde global e sustentabilidade. Cad Saúde Pública 2020; 36:e00040620.
32. Halperin DT. Coping with COVID-19: learning from past pandemics to avoid pitfalls and panic. Glob Health Sci Pract 2020; 8:155-65.

33. Scherer C, Scherer A. Diagnosing and tracking COVID-19 infections leveraging next-gen. sequencing. https://www.thejournalofpreci sionmedicine.com/diagnosing-and-trackingcovid-19-infections-leveraging-next-gen-se quencing/ (acessado em 24/Jun/2020).

34. Reynolds MW, Christian JB, Mack CD, Hall N, Dreyer NA. Leveraging real-World data for COVID-19 research: challenges and opportunities. https://www.thejournalofprecision medicine.com/leveraging-real-world-datafor-covid-19-research-challenges-and-oppor tunities/ (acessado em 24/Jun/2020).

35. Kekic A, Asudani D. COVID-19 and pharmacogenomics: an association that warrants comprehensive assessment. J Precis Med 2020; 2. https://www.thejournalofprecisionmedicine. com/covid-19-and-pharmacogenomics-anassociation-that-warrants-comprehensiveassessment/.

36. Loewy Z. The journey to a COVID-19 diagnostict test. J Precis Med 2020; 2. https:// www.thejournalofprecisionmedicine.com/ the-journey-to-a-covid-19-diagnostic-test/.

37. Power B. How cancer patients should respond to the COVID-19 pandemic. J Precis Med 2020; 2. https://www.thejournalofprecision medicine.com/how-cancer-patients-shouldrespond-to-the-covid-19-pandemic/.

38. Feng J. Retrospect and prospect of precision medical sciences. Precis Med Sci 2020; 9:3.

39. Ferreira AO, Polonini HC, Dijkers ECF. Postulated adjuvant therapeutic strategies for COVID-19. J Pers Med 2020; 10:80.

40. Wang M, Zhou Y, Zong Z, Liang Z, Cao Y, Tang $\mathrm{H}$, et al. A precision medicine approach to managing 2019 novel coronavirus pneumonia. Precis Clin Med 2020; 3:14-21.

41. Jia H, Wu M. Sustained research fund and dedicated research center for preparing next pandemic. Precis Clin Med 2020; [Online ahead of print].

42. Thierry, A H. Host/genetic factors associated with COVID-19 call for precision medicine, Precis Clin Med 2020; 3:228-34.

43. Khoury MJ. From precision medicine to precision public health: beyond the pandemic. https://blogs.cdc.gov/genomics/2020/06/19/ from-precision-medicine/ (acessado em 27/ Jun/2020).

44. Clyne M, Chambers DA, Khoury MJ. Toward more precision in implementation science in the age of COVID-19. https://blogs.cdc.gov/ genomics/2020/06/26/toward-more-preci sion/ (acessado em 10/Jul/2020).

45. Khoury MJ, Gwinn M, Duggal P. The public health impact of COVID-19: why host genomics? https://blogs.cdc.gov/genom ics/2020/04/21/the-public-health-impact/ (acessado em 10/Jun/2020). 
46. Khoury MJ, Bowen S, Braun P. Using digital technologies in precision public health: COVID-19 and beyond. https://blogs.cdc.gov/ genomics/2020/04/06/using-digital/ (acessado em 18/Abr/2020).

47. Vicente AM, Ballensiefen W, Jönsson JI. How personalised medicine will transform healthcare by 2030: the ICPerMed vision. J Transl Med 2020; 18:180.

48. Patel K, Clinton N, Mukhi H, Patel M. COVID-19 presents an opportunity for precision medicine to play expanded role in care. Targeted Therapies in Oncology 2020; 9(5). https://www.targetedonc.com/view/covid19-presents-an-opportunity-for-precisionmedicine-to-play-expanded-role-in-care.

49. Kent J. "All of us" precision medicine program to collect COVID-19 data. https://healthita nalytics.com/news/all-of-us-precision-medi cine-program-to-collect-covid-19-data (acessado em 21/Ago/2020).

50. Shrestha GS, Paneru HR, Vincent JL. Precision medicine for COVID-19: a call for better clinical trials. Crit Care 2020; 24:282.

51. Health Europa. AI precision medicine mining finds 13 human COVID-19 risk genes. https:// www.healtheuropa.eu/ai-precision-medi cine-mining-finds-13-human-covid-19-riskgenes/99851/ (acessado em 23/Ago/2020).
52. World Economic Forum. Global leaders unlock ways for precision medicine to fight COVID-19, cancers and emerging infectious diseases. https://www.weforum.org/ press/2020/05/global-leaders-unlock-waysfor-precision-medicine-to-fight-covid19-cancers-and-emerging-infectious-diseas es/ (acessado em 02/Jun/2020).

53. Personalized Medicine Coalition. COVID-19 and personalized medicine: current status and lessons learned. http://www.personal izedmedicinecoalition.org/Events/Events/ COVID19_and_Personalized_Medicine_ Current_Status_and_Lessons_Learned (acessado em 03/Set/2020).

54. Zimmer C. Dna inherited from Neanderthals may increase risk of COVID-19: the stretch of six genes seems to increase the risk of severe illness from the coronavirus. The New York Times 2020; 4 jul. https://www.nytimes. com/2020/07/04/health/coronavirus-nean derthals.html.

55. Estudo genético pretende mapear grupos de risco da COVID-19. Jornal da USP 2020; 29 mai. https://jornal.usp.br/ciencias/estudo-ge netico-pretende-mapear-grupos-de-risco-dacovid-19/. 


\section{Abstract}

Precision medicine can be defined as a movement of transformation of contemporary biomedicine that orients academic research activity, business models, and the development of health products and services designed individually for the user, based on patients' genetic information and other biomedical markers. In recent years, this community has been quite active in the international scientific scenario. However, during the COVID-19 pandemic, it is still not clear which positions or strategies these groups have adopted to respond to the health crisis. This article aims to understand how the international precision medicine community is reacting to the COVID-19 pandemic and the basis for their approaches and potential solutions suggested for mitigation of the negative effects from the increase in SARS-CoV-2 infections. A search was thus conducted in 28 documents from 18 selected sources, analyzing the narratives adopted by precision medicine experts in scientific articles, editorials, commentaries, perspectives, newspaper stories, newsletters and online conferences of the Personalized Medicine Coalition $(P M C)$. The objective was to understand how these groups envisage a new sociotechnical configuration to respond to the pandemic and its effects.

Precision Medicine; Science, Technology and Society; Health Research Agenda; COVID-19

\section{Resumen}

La medicina de precisión puede definirse como un movimiento de transformación de la biomedicina contemporánea, que orienta la actividad de investigación académica, modelos de negocio y desarrollo de productos, así como de servicios de salud diseñados individualmente para el paciente, basados en información genética y otros marcadores biomédicos de los pacientes. A lo largo de los últimos años, esta comunidad ha sido bastante activa en el escenario científico internacional. No obstante, durante la pandemia de la COVID-19 todavía no quedó claro cuáles son las posiciones o estrategias que han sido adoptadas por esos grupos para enfrentarse a la crisis sanitaria. El objetivo de este ensayo es comprender cómo la comunidad internacional de la medicina de precisión está reaccionando a la pandemia de la COVID-19, y en qué están basados sus abordajes y potenciales soluciones, sugeridos para la mitigación de los efectos negativos causados por el aumento de las infecciones por el nuevo coronavirus. Para ello, se realizó una investigación documental sobre 28 documentos provenientes de 18 fuentes seleccionadas, donde se analizaron las narrativas difundidas por los profesionales de la medicina de precisión en artículos científicos, editoriales, comentarios, perspectivas, noticias de periódicos y boletines, además de la conferencia virtual de la Coalición de Medicina Personalizada (PMC, en inglés). Con todo ello, se buscó comprender cómo esos grupos imaginan una nueva configuración sociotécnica para enfrentarse a la pandemia y sus efectos.

Medicina de Precisión; Ciencia, Tecnología y Sociedad; Agenda de Investigación en Salud; COVID-19
Recebido em 14/Out/2020

Versão final reapresentada em 22/Jan/2021

Aprovado em 26/Jan/2021 